\title{
Tracheal extramedullary plasmacytoma: a rare cause of chronic cough
}

\author{
Rosaria Carrinola 1 , FiorPaolo Basile ${ }^{2}$, Marta Aliprandi², Stefano Bellone ${ }^{3}$, Fulvia Milena Cribiù ${ }^{4}$, \\ Alessandro Vismara ${ }^{2}$, Pierluigi Aliprandi ${ }^{5}$ \\ ${ }^{1}$ UOC Thoracic and Lung Transplant Surgery, IRCCS Cà Granda Ospedale Maggiore Policlinico di Milano; ${ }^{2}$ UOC \\ General Medicine, Rho Hospital, ASST Rhodense, Rho (MI); ${ }^{3}$ UOC Pathological Anatomy, Rho Hospital, ASST \\ Rhodense, Rho (MI); ${ }^{4}$ UOC Pathological Anatomy, IRCCS Cà Granda Ospedale Maggiore Policlinico di Milano, \\ Milan; ${ }^{5}$ Pneumology Clinic, IRCCS Istituto Auxologico Italiano, Milan, Italy
}

\section{Introduction}

Chronic cough and dyspnea are a common reason for medical consultation, but in some cases the cause is to be found in very rare pathologies [1-3]. A 74-year-old, heavy smoker man came to our pulmonary clinic after 3 months of chronic cough and dyspnea. Chest X-ray only showed diffuse interstitial thickening,

Correspondence: Dr. Pierluigi Aliprandi, Pneumology Clinic, IRCCS Istituto Auxologico Italiano, Milano, Italy. Mobile +39.338.4990355. E-mail pierluigi.aliprandi@fastwebnet

Key words: Extramedullary tracheal plasmacytoma; operative rigid bronchoscopy; diode laser; chronic cough.

Authors' contributions: RC (thoracic surgeon), FB (pulmonologist), PA (pulmonologist), performed flexible bronchoscopy and operative rigid bronchoscopy laser assisted; SB (pathologist), performed flexible bronchoscopy biopsy histologic examination; FMC (pathologist), performed tracheal mass histologic examination; AV, performed hematologic evaluation and follow up; MA, (medical student), manuscript drafting and editing. All the authors have read and approved the final version of the manuscript and agreed to be accountable for all aspects of the work.

Conflict of interest: The authors declare that they have no competing interests, and all authors confirm accuracy.

Ethics approval and consent to participate: No ethical committee approval was required for this case report by the Department, because this article does not contain any studies with human participants or animals. Informed consent was obtained from the patient included in this study.

Acknowledgements: We thank Dr Gianfranco Tassi for his scientific support and collaboration and Dr. Vittoria Dentes for editing.

Received for publication: 2 August 2020.

Accepted for publication: 18 September 2020.

${ }^{\circ}$ Copyright: the Author(s), 2020

Licensee PAGEPress, Italy

Monaldi Archives for Chest Disease 2020; 90:1531

doi: 10.4081/monaldi.2020.1531

This article is distributed under the terms of the Creative Commons Attribution Noncommercial License (by-nc 4.0) which permits any noncommercial use, distribution, and reproduction in any medium, provided the original author(s) and source are credited. whilst spirometry showed normal dynamic volumes with notes of distal obstruction. After 3 months of ICS-LABA therapy, cough and dyspnea persisted. Computed tomography scan (CT) and flexible bronchoscopy (FB) showed a $15 \mathrm{~mm}$ diameter endotracheal nodular neoformation. FB biopsy was suggestive for B cell lymphoproliferative pathology. We performed an operative rigid bronchoscopy laser assisted (ORBLA) with endoscopic loop polypectomy preceded and followed by applications of diode laser [4,5] obtaining lesion exeresis with tracheal patency. The histologic examination showed that this lesion was a rare extramedullary plasmacytoma (EMP). The EMP, which constitutes around $4 \%$ of all plasmacell neoplasm, very rarely involve the trachea (only 19 cases were reported until now in medical literature) [1-3,6-11]. CT scan at 14 days and FB control at 20 days and 60 days after ORBLA demonstrated tracheal patency and the biopsy showed no neoplastic infiltration. FB at 5 and 9 months after ORBLA showed complete tracheal patency. The patient was not treated with radiotherapy and at the 1-year follow-up we did not find any recurrences. After that, the patient did not come for any other checkups. In the past days (July 2020), three years after surgery, we have contacted the patient who reported full health.

\section{Case Report}

A 74-year-old, heavy smoker man came to our Institute suffering from chronic coughing and dyspnea for 3 months. At the clinical evaluation, we found vesicular murmur and rhonchi. Chest Xray (dated March 22, 2017) showed only diffuse interstitial thickening (Figure 1) and spirometry showed normal dynamic volumes with notes of distal obstruction. We started ICS-LABA therapy, but after 3 months cough and dyspnea persisted. We then performed a computed tomography scan (CT) (May 5, 2017) which showed a $15 \mathrm{~mm}$ diameter endotracheal nodular neoformation in pars membranacea of medium trachea (Figure 2). FB showed in the middle tracheal third a pink lesion of about $15 \mathrm{~mm}$, with implantation on the pars membranacea, that caused subtotal tracheal occlusion (Figure 3). Lesion's biopsy performed in FB was suggestive for B lymphoproliferative pathology (CD20+; CD3-; CD5-; CD10-). We performed operative rigid bronchoscopy laser assisted (ORBLA) on June 16, 2017 with endoscopic loop polypectomy preceded and followed by applications of diode laser, obtaining lesion exeresis with subtotal tracheal patency (Figure 4) [4,5]. The histological examination of the lesion demonstrated diffuse plasmacell infiltration of chorion mucosae (CD138+; MUM+; CD20+/-; CD19; PAX20-) with intermediate 
degree cytological clonal atypia (chains kappa+, lambda-) and a proliferative index (Ki67) of $10 \%$, leading to the diagnosis of extramedullary plasmacytoma (Figure 5) [3]. Margins of the lesion were not disease-free. After ORBLA, a clinical improvement with cough regression was obtained.

We performed a CT scan 14 days after surgery (June 30, 2017), which showed subtotal tracheal patency (Figure 6). Bone marrow biopsy was negative. Positron emission tomography (PET) showed fluorodeoxyglucose (FDG) uptake only in the peritracheal tissue in correspondence of the previous lesion. Hematological evaluation did not give any indications for medical treatment [12,13]. Radiotherapy evaluation gave indication for adjuvant radiotherapy (RT) $[2,3,13]$ but the patient refused the treatment.

Flexible bronchoscopy control at 20 and 60 days after ORBLA demonstrated subtotal tracheal patency and the biopsy showed no signs of neoplastic infiltration. Five months after ORBLA, PET did not show any tracheal FDG's uptake. Flexible bronchoscopy 9 months after ORBLA showed complete tracheal patency. At the 1year follow-up, we did not find any recurrences. The patient did not show-up at the subsequent follow-ups previously scheduled. In July 2020, three years after surgery, the patient was contacted by Rho Hospital ASST Rhodense's hematology clinic and reported subjective well-being.

\section{Discussion}

Among the possible causes of cough and dyspnea, in addition to the most common pathologies of the respiratory system, tracheobronchial tumors must be taken into consideration. The lack of efficacy of ICS-LABA-LAMA therapies, often used with dubious indications, should suggest the possible presence of a disease quite different from asthma and COPD. Tracheal tumors are not frequent as they constitute around $1 \%$ to $2 \%$ of all respiratory tract tumors $[3,14]$. Among these, squamous cell carcinoma and adenoid

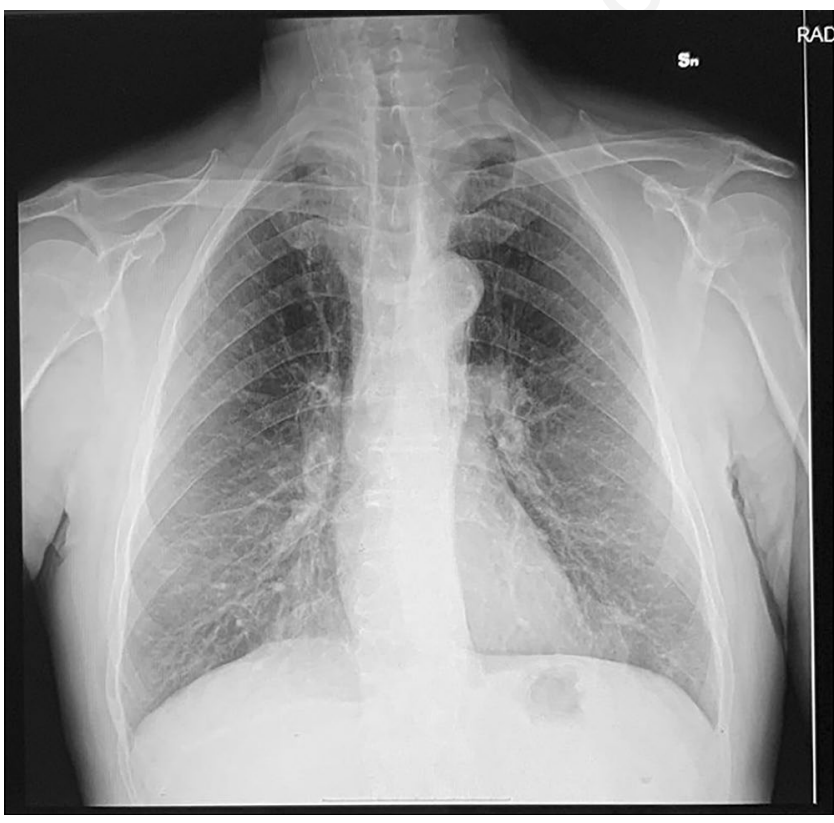

Figure 1. Chest X-ray showed only diffuse interstitium thickening. cystic carcinoma are the most common malignant tumors. EMP represents around $3 \%$ of malignant tracheal tumors $[3,15,16]$. The pathogenesis of tracheal EMP is still unclear, but viral pathogenesis and chronic irritation may contribute to those lesions [3].

EMP are rare plasmacell tumors that arise outside of the bone marrow. They constitute around $4 \%$ of all plasmacell neoplasms, and in $80 \%$ of cases they occur in the upper aerodigestive tractnasal cavity, paranasal sinuses, and oronasopharynx [2,6,15,17]. Only 19 cases were reported until now in medical literature review: Duarte et al. [1] reported 15 cases of tracheobronchial plasmacytoma with tracheal location in 2015 , and the remaining 4 were single case reports [2,3,7-11]. Ours is the twentieth case.

As other tracheal tumors, tracheal plasmacytoma becomes symptomatic when the narrowing of the tracheal lumen is over $75 \%$, as it was in the reported case. Symptoms are nonspecific such as chronic cough, stridor, dyspnea, expiratory wheezing, hoarseness, and hemoptysis. They are often misdiagnosed as asthma or
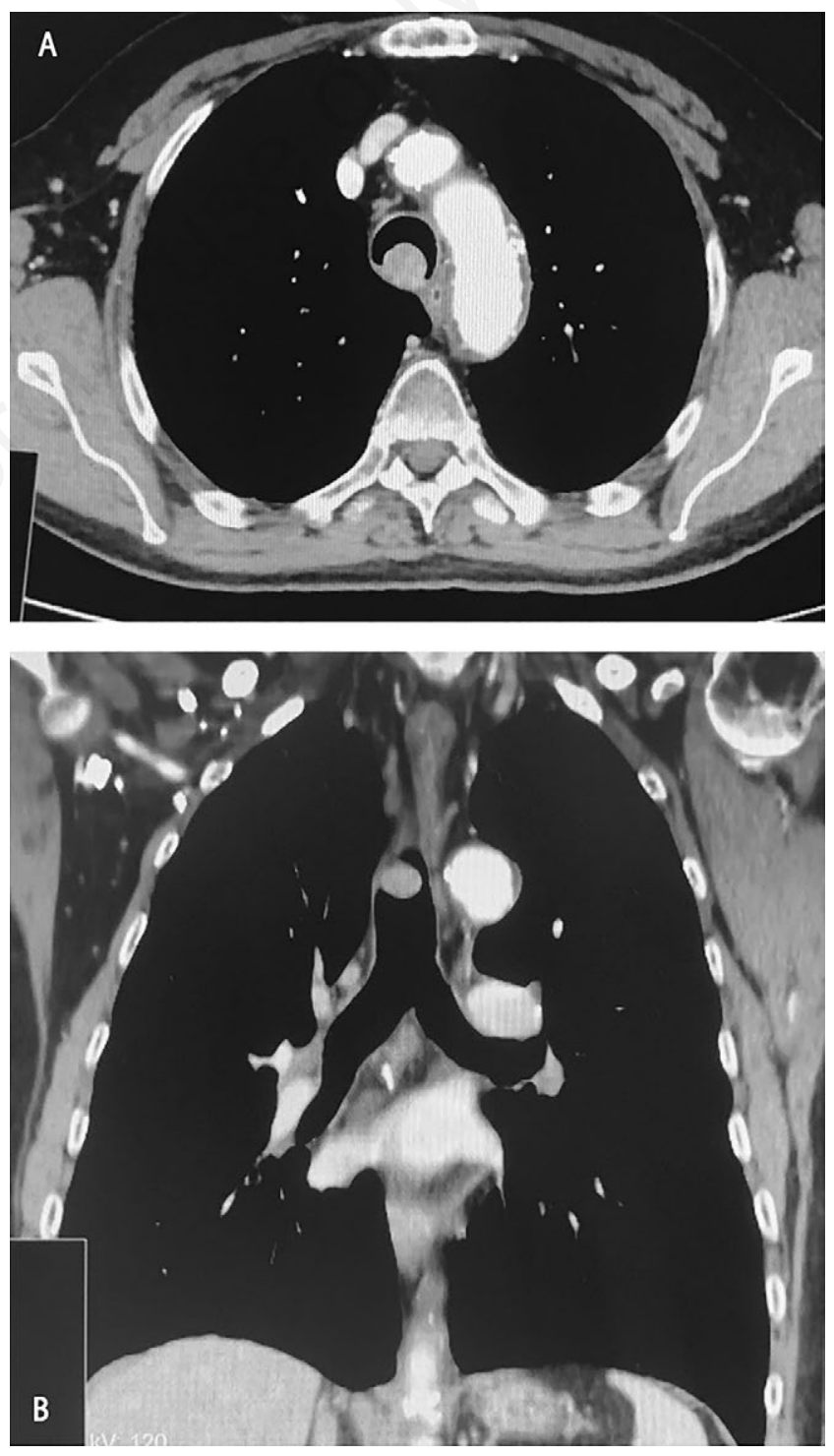

Figure 2. Computed tomography scan revealing $15 \mathrm{~mm}$ diameter endotracheal nodular neoformation in pars membranacea of medium trachea. 
sleep apnea syndrome $[2,6,17]$. Both CT scan and FB are essential diagnostic approaches: whilst the first allows the evaluation of the lumen, airway wall, and mediastinal structures, the second gives an endoluminal view of the tumor [2,3]. There are many options for treatment: surgical resection alone, endoscopic resection alone, RT alone or in combination with one of the first two options

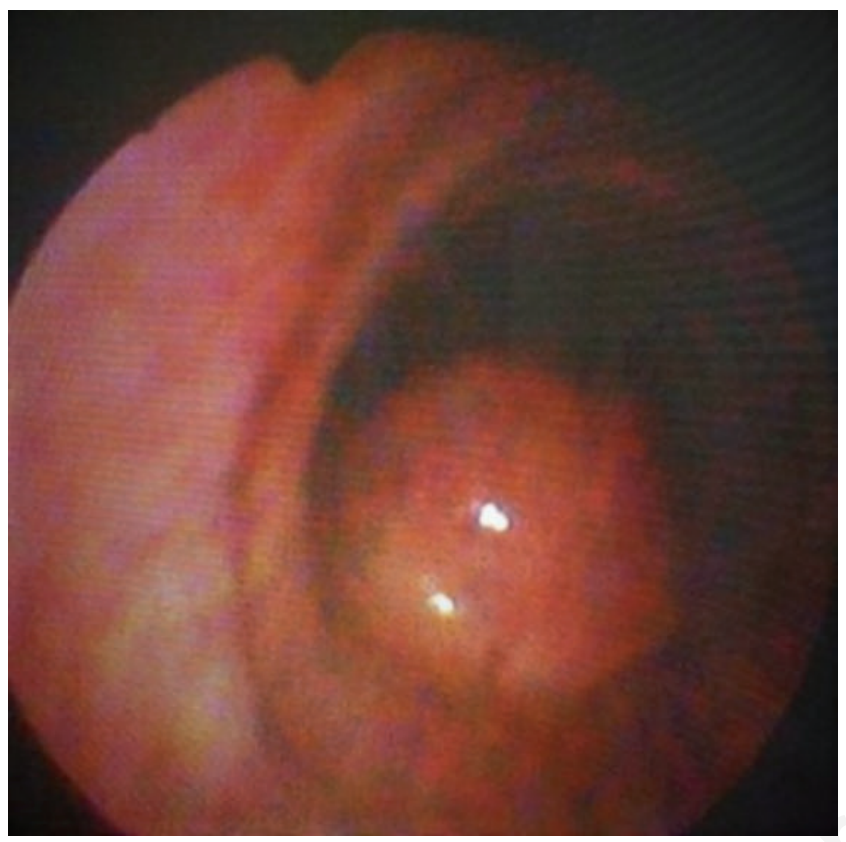

Figure 3. Flexible bronchoscopy confirmed $15 \mathrm{~mm}$ diameter endotracheal nodular neoformation in pars membranacea of medium trachea.

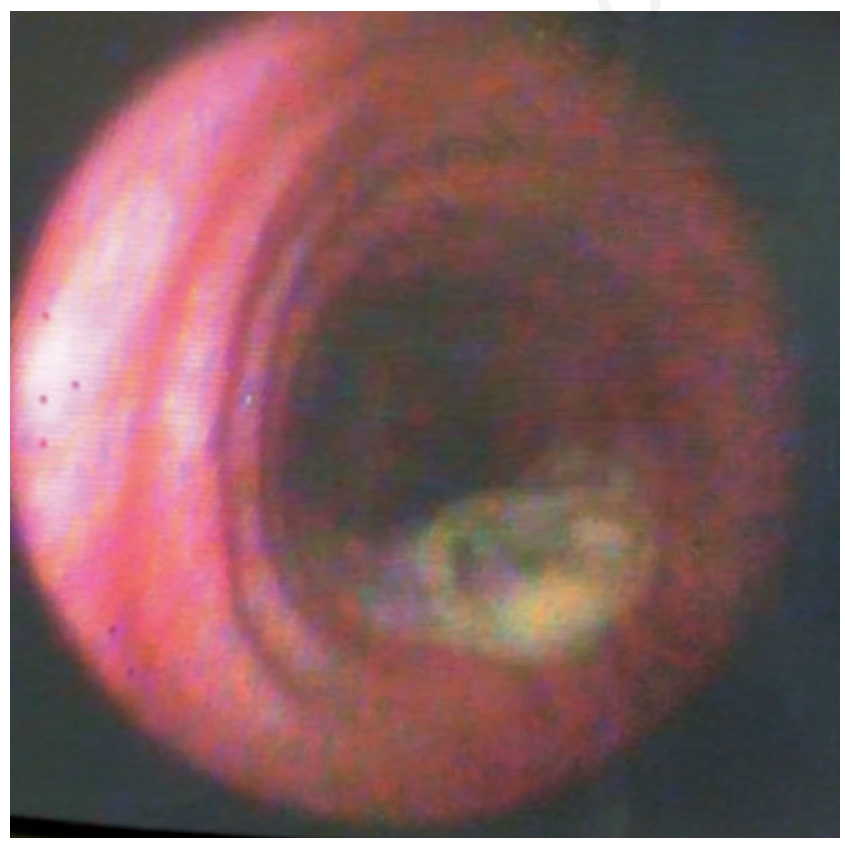

Figure 4. Endoscopic picture after loop polypectomy and diode laser treatment in operative rigid bronchoscopy laser assisted.
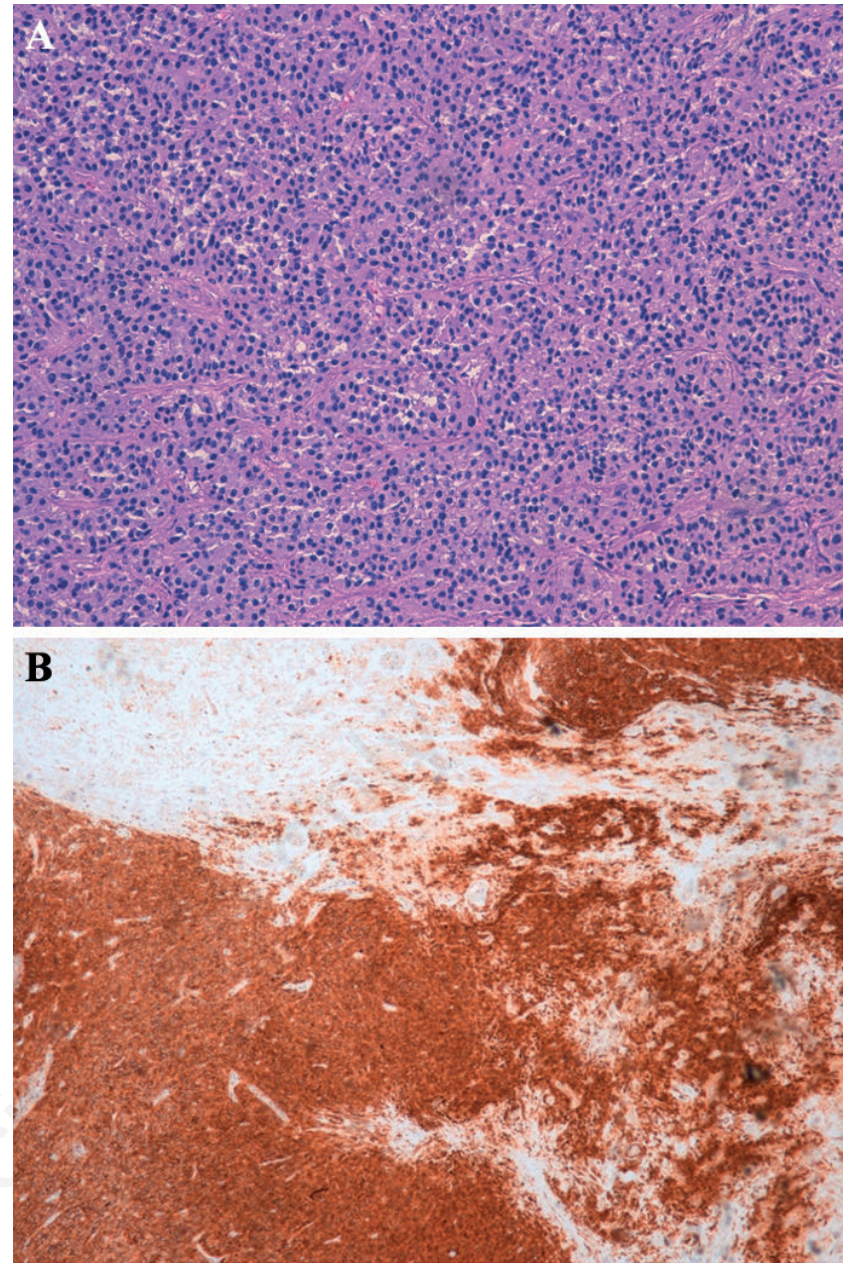

Figure 5. Hematoxylin \& Eosin 20X (A) and CD138 5X (B): diffuse plasmacell infiltration of chorion mucosae (CD138+; MUM+; CD20+/-; CD19; PAX20-) with intermediate degree cytological clonal atypia (chains kappa+, lambda-) and a proliferative index (Ki67) oxyline Ef $10 \%$, leading to the diagnosis of extramedullary plasmacytoma.

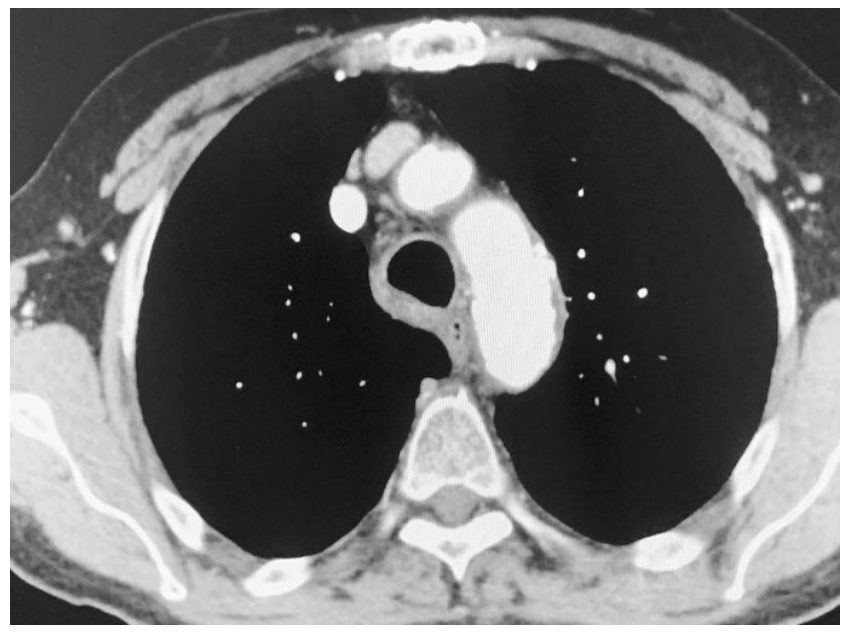

Figure 6. CT showing trachea's patency 14 days after ORBLA treatment. 
$[1,6,13,15]$. There is local recurrence in approximately $30 \%$ and metastasis in $15-40 \%$ of cases $[1,6]$.

In our case, the patient was treated successfully by ORBLA with endoscopic loop polypectomy, diode laser treatment, and no RT $[4,5]$. Diode laser treatment of the lesion before removal and of the lesion's base after removal were likely effective. During the procedure no bleeding occurred, and even if lesion's margins were not disease free, at the 1-year follow-up no signs of recurrences have been observed. FB control at 20 days, 60 days, 5 months, 9 months after ORBLA demonstrated tracheal patency and no neoplastic infiltration in biopsy. After one year, the patient failed to attend clinical checks. But, in July 2020, three years after operation, the patient was contacted by Hematology Clinic and reported subjective well-being.

\section{Conclusions}

Tracheal tumors account for only $1-2 \%$ of all respiratory tract tumors $[1,2,15]$. EMP are rare plasmacell tumors that arise outside of the bone marrow. They constitute only around $4 \%$ of all plasmacell neoplasms. Although most of EMP arise in upper airways, they are rarely present in trachea $[2,6,15,17]$. Only 19 cases were reported until now in medical literature review [1-3,7-11] and ours is the twentieth case. Symptoms are nonspecific such as chronic cough, dyspnea and expiratory wheezing and are often misdiagnosed as asthma or COPD [3]. CT and FB are an essential diagnostic tool $[2,3]$. Regardless of treatment options, the low number of cases reported in literature does not allow for an evidence-based approach to EMP treatment. Surgery/endoscopic resection and/or chemoradiation remain controversial [3].

In our case, after a bronchofibroscopy that did not lead to a definitive histological diagnosis, we have decided to submit the patient to an ORBLA. This technique, which involves less morbidity than tracheal surgery, led to the prompt regression of symptoms (dyspnea and cough), to the correct histological definition of EMP, and allowed us to carry out the laser treatment of the neoplasm. The patient refused the proposed radiotherapy, and the absence of the disease's recurrence observed in the one-year follow-up is likely to be related to the effectiveness of the ORBLA and, in particular, to the laser treatment performed on the neoplasm and its implantation base.

In light of this clinical case, it is possible to hypothesize that ORBLA, as reported in the literature for benign tracheobronchial tumors, carcinoma in situ, minimally invasive endobronchial lesions and typical carcinoid $[5,18]$ can have a radical curative role in the rare EMP of the trachea in carefully selected and wellinformed patients.

\section{References}

1. Duarte ML, de Brito MC, Traple FA et al. Plasmacytoma of the trachea: a surprising diagnosis. Radiol Bras 2017;50:66-7.

2. Stevic R, Ercegovac M, Stojšić J, Čolić N. Rare tracheal tumor: Solitary plasmacytoma. J Postgrad Med 2018;64:115-8.

3. Zhang X, Su L, Ran YG, Qie S et al. Extramedullary plasmacytoma of the trachea: A case report and review of the literature. Medicine (Baltimore) 2018;97:e9594.

4. Du Rand IA, Barber PV, Goldring J, et al. British Thoracic Society guideline for advanced diagnostic and therapeutic flexible bronchoscopy in adults. Thorax 2011;66:iii1 eiii21.

5. Cavaliere S, Milani GF. Laserterapia endoscopia bronchiale terapeutica. In: Casalini A, Editor. Pneumologia Interventistica. Springer: 2007. p. 362-75.

6. Kairalla RA, Carvalho CR, Parada AA, et al. Solitary plasmacytoma of the trachea treated by loop resection and laser therapy. Thorax 1988;43:1011-2.

7. Jizzini MN, Shah M, Yeung SJ. Extramedullary plasmacytoma involving the trachea: A case report and literature review. J Emerg Med 2019;57:e65-7.

8. Sukumaran R, Nair RA, Jacob PM et al. Extramedullary plasmacytoma of the trachea. Head Neck Pathol 2014;8:220-4.

9. Cuttitta A, Tancredi A, Scaramuzzi R, et al. Solitary extramedullary plasmacytoma of the trachea: A case report. Int J Cardiovasc Thorac Surg 2016;2:5-8.

10. Durris N, Rosnet G, Clemenson A, Vergnon JM. Characteristics and management of tracheobronchial extramedullary plasmacytomas. J Bronchology 2002;9:186-92.

11. Dines DE, Lillie JC, Henderson LL, et al. Solitary plasmacytoma of the trachea. Am Rev Respir Dis 1965;92:949-51.

12. Soutar R, Lucraft H, Jackson G, et al. Guidelines on the diagnosis and management of solitary plasmacytoma of bone and solitary extramedullary plasmacytoma. $\mathrm{Br} \mathrm{J}$ Haematol 2004; $124: 717$.

13. Wang K, Huang Y, Chen A. A case report of tracheal extramedullary plasmacytoma. Chin J Clin Oncol 2008;5:311-2.

14. Ferretti GR, Bithigoffer C, Righini CA, et al. Imaging of tumors of the trachea and central bronchi. Thorac Surg Clin 2010;20:31-45.

15. Garelli M, Righini C, Faure C, et al. Imaging of a case of extramedullary solitary plasmacytoma of the trachea. Case Rep Radiol 2011;2011:687203.

16. Rai SP, Kumar R, Bharadwaj R, et al. Solitary tracheal plasmacytoma. Indian J Chest Dis Allied Sci 2003;45:269-72.

17. Kober SJ. Solitary plasmacytoma of the carina. Thorax. 1979;34:567-8.

18. Carlata S, Fuso L, Galluccio G. The technique of endoscopic airway tumor treatment. J Thorac Dis 2017;9:2619-39. 